\title{
Von Sekundärwahlen zu europäisierten Wahlen? 30 Jahre Direktwahlen zum Europäischen Parlament
}

\author{
Jürgen Mittag und Claudia Hülsken*
}

Die Debatten über die Direktwahlen des Europäischen Parlaments reichen historisch bis in die Anfangsjahre des europäischen Integrationsprozesses zurück. ${ }^{1}$ Es sollte jedoch bis zum (Rats-)Beschluss und Akt zur Einführung allgemeiner unmittelbarer europäischer Wahlen am 20. September 1976 dauern, bis sich die Mitgliedstaaten der Gemeinschaft darauf verständigten, dass nationale Abgeordnete nicht mehr als Doppelmandatäre ins Europäische Parlament delegiert werden, sondern die Parlamentarier ihre Legitimation fortan aus der Direktwahl durch die Bevölkerung beziehen. ${ }^{2}$ Die Erwartungen, die in die ersten Direktwahlen gesetzt wurden, waren beträchtlich, wie exemplarisch ein Redeausschnitt von Hans-Dietrich Genscher aus dem Jahr 1977 dokumentiert: „Mit der Direktwahl zum Europäischen Parlament wird“, so erklärte der Bundesaußenminister vor dem Deutschen Bundestag, ,ein neuer Abschnitt in der Geschichte der europäischen Einigung beginnen. [...] Zum ersten Mal wird ein Parlament entstehen, das europäisch legitimiert ist. [...] Der Wahlkampf selbst wird das Thema Europa endgültig aus den Konferenzsälen der Experten und Regierungen heraustragen und zu den Bürgern bringen und ihnen bewusstmachen, wie sehr ihre eigene Zukunft mit der Zukunft der Gemeinschaft verbunden ist. " ${ }^{3}$ In dieser knappen Redepassage spiegeln sich zahlreiche Motive wider, die in den 1970er Jahren mit der Einführung von Direktwahlen verbunden worden waren, ${ }^{4}$ in den folgenden drei Dekaden aber nur in begrenztem Maße zum Tragen kamen.

Vom 4. bis zum 7. Juni 2009 wird die mittlerweile siebte Direktwahl zum Europäischen Parlament stattfinden. Nach dem Beitritt Bulgariens und Rumäniens sind rund 375 Millionen Europäer - und damit mehr Unionsbürger als jemals zuvor - zum gemeinsamen Urnengang in Europa aufgerufen. Die bevorstehende Europawahl nimmt dieser Beitrag zum Anlass, eine knappe Bilanz des Forschungsstands zu Europawahlen zu ziehen und zugleich einen historischen Rückblick auf die empirischen Trends bei den Direktwahlen der vergangenen 30 Jahre vorzunehmen.

Mit dieser diachronen Perspektive soll vor allem der Frage nach dem Wandel beziehungsweise der Konstanz von Europawahlen nachgegangen werden. Obwohl hierbei die deutsche Perspektive im Vordergrund steht, werden in der folgenden - auf insgesamt fünf Problemfelder konzentrierten - Standortbestimmung auch Entwicklungen in den anderen

* Dr. Jürgen Mittag, Geschäftsführer des Instituts für soziale Bewegungen, Ruhr-Universität Bochum.

Claudia Hülsken, B.A., Wissenschaftliche Hilfskraft am Institut für soziale Bewegungen, Ruhr-Universität Bochum.

1 Vgl. zu den langwierigen Kontroversen über die Direktwahl Gerhard Brunn: Das Europäische Parlament auf dem Weg zur ersten Direktwahl 1979, in: Franz Knipping/Matthias Schönwald (Hrsg.): Aufbruch zum Europa der zweiten Generation. Die europäische Einigung 1969-1984, Trier 2004, S. 47-72 und Holger-Michael Arndt/Johannes Eißer: 25 Jahre Direktwahlakt - Die Wahlrechte zum Europäischen Parlament, Berlin 2004.

2 Den Akt selbst kennzeichnet rechtlich eine hybride Qualität zwischen EG-Sekundärrecht und Primärrecht, da er nach der Verabschiedung im Rat noch den Mitgliedstaaten zur Zustimmung vorgelegt wurde.

3 Rede von Hans-Dietrich Genscher vor dem Deutschen Bundestag am 26. Mai 1977, in: Plenarprotokoll des Bundestages 8/29 vom 26.05.1977, S. 2035 (D)-2037 (B).

4 Vgl. zu den Motiven vor allem Institut für Europäische Politik (Hrsg.): Die erste Direktwahl des Europäischen Parlaments. Motive, Wahlkampf, Resultate und Perspektiven, zusammengestellt und eingeleitet von Emanuel Richter, Bonn 1981. 
EU-Mitgliedstaaten berücksichtigt: Nach einem knappen Überblick zur Europawahlforschung werden, erstens, die Kampagnen und medialen Kontexte, die den Europawahlkampf prägten, behandelt; zweitens werden die Kontroversen über die Wahlsysteme bei Europawahlen aufgegriffen; drittens wird die Entwicklung der Wahlbeteiligung beleuchtet; eine Analyse der Wahlresultate bildet das vierte Problemfeld, dem sich, fünftens, eine Betrachtung der Fraktionsbildung im Europäischen Parlament anschließt. Mit diesem breit gesteckten Themenspektrum zielt der Beitrag weniger auf eine Wahlanalyse im Sinne der quantifizierenden Auswertungen einer zunehmend theoretisch und methodisch ausdifferenzierten Wahlforschung, ${ }^{5}$ als vielmehr auf die Betrachtung grundlegender Entwicklungslinien von Europawahlen seit 1979. Gerichtet ist der Blick dabei insbesondere auf die Frage, inwieweit die Europawahlen einem Trend zur Europäisierung unterliegen. Der vielschichtige Europäisierungsbegriff wird in diesem Zusammenhang in einem eher heuristischen Verständnis als Anpassungsprozess verwendet, der im Sinne des Diktums von Hans-Dietrich Genscher eine Stärkung der europäischen Dimension von Europawahlen beziehungsweise eine Schwächung ihrer Perzeption als nationale Sekundärwahlen von nachrangiger Bedeutung unterstellt. ${ }^{6}$

\section{Europawahlen in der wissenschaftlichen Forschung: Dominanz der Momentauf- nahmen}

Die wissenschaftliche Forschung zu Europawahlen zeichnet sich durch die Dominanz von Einzelfallstudien sowie eine lediglich geringe Anzahl von übergreifenden oder systematisch vergleichenden Darstellungen aus. Wissenschaftliche Analysen kamen bisher in erster Linie aus den Reihen der Sozialwissenschaften, im Rahmen der Wahlrechtsdebatte haben aber immer wieder auch Juristen wichtige Beiträge beigesteuert. ${ }^{7}$ Grundlegend lässt sich zwischen drei Strängen der Europawahlforschung unterscheiden. Ein erster, durch zahlreiche normative Forschungsarbeiten inspirierter Strang umspannt die Phase zwischen der Vorbereitung und Nachbetrachtung der ersten Direktwahl. In diesem Zusammenhang sind vor allem die rechtlichen und institutionellen Rahmenbedingungen der Direktwahl eingehender bearbeitet worden. Neben Untersuchungen zum Wahlsystem und Wahlrecht ${ }^{8}$ standen dabei vor allem die transnationalen Parteienkontakte und die Formierung von europäischen Parteiorganisationen ${ }^{9}$ sowie die Verabschiedung gemeinsamer Wahlplattformen und -manifeste im Blickfeld. ${ }^{10}$ Bei den eigentlichen Wahlanalysen dominierte 1979 die Frage, inwie-

5 Vgl. hierzu als Überblick Oscar W. Gabriel/Silke I. Keil: Empirische Wahlforschung in Deutschland: Kritik und Entwicklungsperspektiven, in: Jürgen W. Falter/Harald Schoen (Hrsg.): Handbuch Wahlforschung, Wiesbaden 2005, S. 611-641.

6 Siehe hierzu auch Heinz-Jürgen Axt: Wahlen zum Europäischen Parlament: Trotz Europäisierungstendenzen noch immer nationale Sekundärwahlen, in: Claudia Derichs/Thomas Heberer (Hrsg.): Wahlsystem und Wahltypen. Politische Systeme und regionale Kontexte im Vergleich, Wiesbaden 2006, S. 122-144.

7 Unverändert zentral für eine Synthese beider Disziplinen ist Eberhard Grabitz/Otto Schmuck/Sabine Steppat/ Wolfgang Wessels: Direktwahl und Demokratisierung. Eine Funktionsbilanz des Europäischen Parlaments nach der ersten Wahlperiode, Bonn 1988 und Eberhard Grabitz/Thomas Läufer: Das Europäische Parlament, Bonn 1980, vor allem S. 233-292.

8 Vgl. etwa Roland Bieber: Funktion und Grundlagen direkter Wahlen zum Europäischen Parlament im Jahre 1978, in: Zeitschrift für Parlamentsfragen 2/1976, S. 228-244; Peter-Christian Müller-Graff: Die Direktwahlen des Europäischen Parlaments. Genese und Perspektiven, Tübingen 1977.

9 Siehe aus der Vielzahl von Studien im Kontext der ersten Direktwahlen des Europäischen Parlaments beispielhaft Joachim Raschke: Die politischen Parteien in Westeuropa. Geschichte - Programm - Praxis. Ein Handbuch, Hamburg 1978 und Theo Stammen: Parteien in Europa. Nationale Parteiensysteme. Transnationale Parteienbeziehungen. Konturen eines europäischen Parteiensystems, 2. Auflage, München 1978.

10 Vgl. Eva-Rose Karnofsky: Parteienbünde vor der Europa-Wahl 1979. Integration durch gemeinsame Wahlaussagen?, Bonn 1982. 
weit dieses Wahlergebnis Ausdruck einer pro-europäischen Haltung oder Resultat einer europaskeptischen Positionierung von Bevölkerung, Parteien und Staaten sei. ${ }^{11}$

Ein zweiter - bis heute wirksamer - Strang der Europawahlforschung wurde durch Karlheinz Reif, langjähriger Leiter des Referats Meinungsumfragen der Europäischen Kommission, und Hermann Schmitt, Projektleiter am Mannheimer Zentrum für Europäische Sozialforschung, begründet. Beide Wissenschaftler haben bereits im Kontext der ersten Direktwahl 1979 Europawahlen als ,second-order elections“ klassifiziert und damit ein Erklärungsmodell eingeführt, das in der Folge vielfach aufgegriffen wurde und bis heute paradigmatisch für die Analyse von Europawahlen ist. ${ }^{12}$ Im kontrastierenden Vergleich zu nationalen Wahlen - sogenannten ,first-order elections ' - werden Europawahlen als Wahlen ,zweiter Ordnung ' bewertet, die von innerstaatlichen Themen und parteipolitischen Auseinandersetzungen der nationalen Ebene beherrscht werden. ,Second-order elections ‘ zeichnen sich zudem durch eine niedrigere Wahlbeteiligung sowie dadurch aus, dass nationale Regierungsparteien zumeist als ,Verlierer' aus den Direktwahlen zum Europäischen Parlament hervorgehen - vor allem, wenn die Wahlen zur Mitte nationaler Legislaturperioden stattfinden. Das Konzept der Sekundär- oder Nebenwahlen wurde bei den nachfolgenden Europawahlen immer wieder verwendet ${ }^{13}$ und von der Forschung auch weitgehend empirisch bestätigt. Nicht zuletzt durch das Forschernetzwerk im Kontext der „European Election Studies“, ${ }^{4}$ in dessen Rahmen seit 1979 Wahlverhalten, Kandidaten, Wahlprogramme sowie weitere, vor allem quantitative Daten erhoben und analysiert wurden, hat sich der Begriff „,zu einem Passepartout entwickelt, das zur Erklärung aller Besonderheiten [...] von Europawahlkämpfen dient“" und damit, so Christina Holtz-Bacha, ,längst über das hinausgeht, was zunächst damit gemeint war". ${ }^{15}$ Als ein zunehmend an Bedeutung gewinnender Nebenstrang des ,second-order' Konzepts ist die Beschäftigung mit den Ursachen des anhaltenden Rückgangs der Wahlbeteiligung auszumachen. ${ }^{16}$

Ein dritter Strang der Europawahlforschung, der Ansätze aus den frühen 1980er Jahren wieder aufgreift, zeichnet sich seit Beginn des 21. Jahrhunderts ab. Mit Blick auf die zunehmende Beachtung von kommunikativen und medialen Ereignissen wird sowohl dem Euro-

11 Siehe exemplarisch Rudolf Hrbek: Die EG nach den Direktwahlen. Bilanz und Perspektiven, in: integration 3/1979, S. 95-109; Valentine Herman/Mark Hagger (Hrsg.): The Legislation of Direct Elections to the European Parliament, Farnborough 1980.

12 Vgl. Karlheinz Reif/Hermann Schmitt: Nine Second-Order National Elections - A conceptual Framework for the Analysis of European Election Results, in: European Journal of Political Research 1/1980, S. 3-45.

13 Vgl. Karlheinz Reif (Hrsg.): Ten European Elections. Campaigns and Results of the 1979/81 First Direct Elections to the European Parliament, Aldershot 1985, S. 1-36; Pippa Norris: Second-order elections revisited, in: European Journal of Political Research 1-2/1997, S. 109-114; Michael Marsh: Testing the Second-Order Election Model after Four European Elections, in: British Journal of Political Science 4/1998, S. 591-607; Stéphanie Abrial/Christine Pina: Les élections européennes de juin 1999 dans les quinze pays de l'Union: Une consultation de „second ordre“?, in: Revue française de science politique 4/1999, S. 707-717 und Hermann Schmitt: The European Parliament Elections of June 2004: Still Second-Order?, in: West European Politics 3/2005, S. 650-679.

14 Siehe zu diesem Netzwerk, das im Kontext der Europawahlen 2009 vom Europäischen Hochschulinstitut in Florenz aus koordiniert wird, die Internetseite unter: http://www.piredeu.eu (letzter Zugriff: 26.03.2009).

15 Christina Holtz-Bacha: Massenmedien und Europawahlen: low key campaigns - low key response, in: Christina Holtz-Bacha (Hrsg.): Europawahl 2004. Die Massenmedien im Europawahlkampf, Wiesbaden 2005, S. 7 34, hier S. 7.

16 Vgl. Cees van der Eijk/Mark Franklin/Michael Marsh: What Voters Teach Us About Europe-Wide Elections: What Europe-Wide Elections Teach Us About Voters, in: Electoral Studies 2/1996, S. 149-166; Hermann Schmitt/Cees van der Eijk: Die politische Bedeutung niedriger Beteiligungsraten bei Europawahlen. Eine empirische Studie über die Motive der Nichtwahl, in: Frank Brettschneider/Jan van Deth/Edeltraud Roller (Hrsg.): Europäische Integration in der öffentlichen Meinung, Opladen 2003, S. 279-302. 
pawahlkampf als auch den Kampagnen und der medialen Rezeption seitens der Forschung verstärkte Aufmerksamkeit gewidmet, ${ }^{17}$ ohne dass das Konzept der , second-order elections * grundlegend infrage gestellt oder gar aufgegeben wurde. ${ }^{18}$

\section{Europawahlkampagnen, Kandidaten und Medienberichterstattung: vom ,Wunsch- zum Stiefkind}

Im Rahmen von Wahlkämpfen als „Zeiten intensiver politischer Kommunikation“19 werden durch Kommunikationskanäle im Wesentlichen drei Akteursgruppen miteinander verbunden: die nationalen Parteien und ihre (Spitzen-)Kandidaten als politische Aktivisten, ${ }^{20}$ die wahlberechtigte Bevölkerung als primärer Adressat und Rezipient der parteieninitiierten Wahlkampfkommunikation sowie die Massenmedien als vermittelnde Instanzen zwischen der politischen Elite und der wahlberechtigten Bevölkerung. Mit Blick auf dieses Akteursdreieck gehören die Wahlkampagnen der Parteien, die Kandidatenauswahl sowie die Berichterstattung in den Medien zu den konstitutiven Elementen von Wahlkämpfen, die im Folgenden näher beleuchtet werden:

Wahlkampfthemen: Die Wahlkampfthemen der Europawahlen wiesen bei allen bisherigen Wahlen nur in Ansätzen einen transnationalen Charakter auf. Die europäischen Parteienbünde erarbeiten zwar seit der ersten europäischen Direktwahl eigene Wahlprogramme beziehungsweise -aufrufe, die in der Regel einen mühsam zustande gekommenen inhaltlich-programmatischen Konsens widerspiegeln. ${ }^{21}$ Die Wahlkämpfe selbst wurden bisher jedoch primär zwischen den konkurrierenden Parteien der einzelnen Mitgliedstaaten ausgetragen und zeigten eine starke nationale Prägung. Eine viel zitierte Ausnahme stellt lediglich das Parteienbündnis der grünen Parteien im Wahlkampf 2004 dar, das mit dem Slogan „Europe can do better - You decide“ sowie einer gemeinsamen Kampagne aufwartete, an der sich etwa 25 grüne Parteien beteiligten. ${ }^{22}$

17 Vgl. Jens Tenscher (Hrsg.): Wahl-Kampf um Europa. Analysen aus Anlass der Wahlen zum Europäischen Parlament 2004, Wiesbaden 2005 oder Claes H. de Vreese/Susan A. Banducci/Holli A. Semetko/Hajo G. Boomgaarden: The news coverage of the 2004 European Parliamentary Election Campaign in 25 countries, in: Michael Marsh/Slava Mikhaylov/Hermann Schmitt (Hrsg.): European Elections after Eastern Enlargement. Preliminary Results from the European Elections Study 2004, Mannheim 2007, S. 17-52.

18 Siehe hierzu etwa Oskar Niedermayer/Hermann Schmitt (Hrsg.): Europawahl 2004, Wiesbaden 2005; Andreas Maurer/Dietmar Nickel (Hrsg.): Das Europäische Parlament. Supranationalität, Repräsentation und Legitimation, Baden-Baden 2005.

19 Harald Schoen: Wahlkampfforschung, in: Jürgen W. Falter/Harald Schoen (Hrsg.): Handbuch Wahlforschung, Wiesbaden 2005, S. 503-542, hier S. 505.

20 In Bezug auf die Europawahlen sind zudem die europäischen Parteiorganisationen zu nennen, denen aber aufgrund der nationalen Wahlprozedere sowie begrenzter Ressourcen lediglich eine marginale Rolle im Rahmen der europäischen Direktwahlen zukommt. Siehe hierzu auch Clemens zur Hausen: Der Beitrag der „Europarteien“" zur Demokratisierung der Europäischen Union, Marburg 2008, S. 168-197.

21 Vgl. Eva-Rose Karnofsky: Die europäischen Parteienzusammenschlüsse vor der ersten Direktwahl des Europäischen Parlaments. Die Entstehung ihrer programmatischen Aussagen, Tübingen 1981; Cees van der Eijk/ Erik V. Oppenhuis: European parties' performance in electoral competition, in: European Journal of Political Research 1/1991, S. 55-80.

22 In diesem Rahmen wurde ein europaweit einheitliches Erscheinungsbild mit, originär grünen ‘ Themen, einem einheitlichen Logo sowie identischen Motiven und einem Team von Spitzenkandidaten vereinbart. Siehe Andreas von Gehlen: Europäische Parteiendemokratie? Institutionelle Voraussetzungen und Funktionsbedingungen der europäischen Parteien zur Minderung des Legitimationsdefizits der EU. European party democracy?, Dissertation Freie Universität Berlin 2005, S. 299; Yvonne Nasshoven: „Let's Green Europe“? Von der Europäischen Föderation Grüner Parteien zur Europäischen Grünen Partei, in: Jürgen Mittag (Hrsg.): Politische Parteien und europäische Integration. Entwicklung und Perspektiven transnationaler Parteienkooperation in Europa, Essen 2006, S. 617-637, hier vor allem S. 632-633. 
Dass die nationalen Parteien Europawahlkämpfe grundsätzlich weniger mit europäischen Themen als vielmehr mit nationalen - allenfalls oberflächlich mit Europabezügen etikettierten - Themen bestreiten, und dass sich die Rhetorik der Kampagnen bei Plakaten oder Wahlwerbespots auf abstrakte und weitgehend unstrittige Schlagworte (Frieden, Freiheit, Wohlstand, Sicherheit) sowie die Betonung der eigenen Europakompetenz konzentriert, lässt sich für alle bisherigen Europawahlen in Deutschland bestätigen. Dokumentiert wird dies beispielhaft durch die Wahlwerbeslogans der FDP: Von der ersten Europawahl 1979 (,Ja zu Europa“ und „Europa liberal“), über die Wahlkämpfe in den 1980er Jahren (1984: „Ja zu Europa. Nein zum Streik“, „Europa braucht die F.D.P.“; 1989: „Das liberale Europa lohnt sich“) und in den 1990er Jahren (1994: „Im Namen des Wohlstands: Wir brauchen Europa“ sowie 1999: „Gelbe Karte für Rot-Grün“) bis hin zur liberalen Europawahlkampagne 2004 („Wir können Europa besser“) lässt sich - mit Ausnahme von 1999 - kaum ein Wandel bei den Wahlslogans erkennen. Bei den Kampagnen der anderen Parteien zeichnet sich ein ähnliches Bild ab, das in diachroner Perspektive ebenfalls kaum Veränderungen aufweist.

Ungeachtet des wiederholten Rekurses auf ,Europa‘ im Allgemeinen, wurden die Wahlkämpfe im Detail aber mit nationalen Themen bestritten. Als zentrale Ursache für die Nationalisierung der Wahlkampfthemen ist sicherlich das - auch durch einen weitgehenden proeuropäischen Grundkonsens ${ }^{23}$ bedingte - fehlende europapolitische Differenzierungspotenzial der deutschen Parteienlandschaft auszumachen; zudem verspricht die Thematisierung innenpolitischer Aspekte eine stärkere Wählermobilisierung. Selbst tief greifende politische Veränderungen auf europäischer Ebene wie die Maastrichter Vertragsrevision Anfang der 1990er Jahre, ${ }^{24}$ der Rücktritt der Europäischen Kommission im Vorfeld der Europawahlen 1999 oder die Debatten um den Verfassungsvertrag und die mittel- und osteuropäische Erweiterung zu Beginn des 21. Jahrhunderts haben die europalose Rhetorik der Wahlkämpfe nicht verändert.

Wahlkampforganisation: Bei nationalen Parlamentswahlkämpfen beziehungsweise ,firstorder elections' lässt sich seit den 1980er Jahren eine zunehmende Professionalisierung der Wahlkampfführung feststellen, die ihren Ausdruck unter anderem in der Verpflichtung hauptamtlicher Kräfte zur Kampagnenführung und -planung sowie dem Engagement professioneller externer Wahlkampfberater findet. Im Zuge dieser Professionalisierung ist auch eine Ausweitung des Kampagnenzeitraums - bis hin zu einer , permanent campaign “ - zu beobachten. Während entsprechende Strategien und Techniken mittlerweile zu festen Bestandteilen des Wahlkampfmanagements der Parteien bei nationalen Wahlen avanciert sind, konstatiert Jens Tenscher für die Europawahlkämpfe eine ,,anachronistische“ Kampagnenentwicklung. ${ }^{25}$ Diese kann an mehreren Merkmalen festgemacht werden: Verglichen mit den nationalen Wahlen zeichnen sich Europawahlkämpfe durch eine relativ kurze Kampagnendauer, einen geringen Grad an Externalisierung wahlkampfspezifischer Aufgaben sowie ein knapperes

23 Eine Abweichung vom pro-europäischen Grundkonsens zeigen nur Anti-EU-Parteien wie die Republikaner. Siehe hierzu auch Tanja Binder/Andreas M. Wüst: Inhalte der Europawahlprogramme deutscher Parteien 1979-1999, in: Aus Politik und Zeitgeschichte B 17/2004, S. 38-45, hier S. 41.

24 Für die Europawahlen 1994 und den Nachrichtenwert der Direktwahlen besonders prägend war die Tatsache, dass im gleichen Jahr Bundestagswahlen stattfanden. Dadurch wurde die nationale Kontextualisierung der Europawahlen begünstigt beziehungsweise eine Transnationalisierung der Parteikampagnen erschwert. Vgl. auch Oskar Niedermayer: Die Europawahlen in der Bundesrepublik im Kontext des Superwahljahres 1994, in: integration 1/1995, S. 22-30, hier S. 22.

25 Vgl. Jens Tenscher: Wahl-Kampf um Europa. Eine Einführung, in: Jens Tenscher (Hrsg.): Wahl-Kampf um Europa. Analysen aus Anlass der Wahlen zum Europäischen Parlament 2004, Wiesbaden 2005, S. 7-30, besonders S. 13-14; Schoen: Wahlkampfforschung, 2005, S. 514. 
Budget aus. Gaben die deutschen Parteien im Rahmen der Bundestagswahlen 2002 knapp 70 Millionen Euro für ihre Wahlkampfkampagnen aus, ${ }^{26}$ investierten sie zwei Jahre später mit 29,2 Millionen Euro weniger als die Hälfte in ihre Werbung zur europäischen Direktwahl.

Eine Kontinuität hinsichtlich der Budgetierung der Europawahlkämpfe kann auch daran festgemacht werden, dass die Einnahmen durch staatliche Wahlkampfkostenerstattung regelmäßig die tatsächlichen Ausgaben der subventionierten Parteien überstiegen. Das Beispiel des Europawahlkampfs 2004 scheint den Vorwurf, die Parteien würden mit der Europawahl ,Gewinn machen', zu bestätigen: Während die bei der Europawahl 2004 in Deutschland angetretenen 24 Parteien und politischen Vereinigungen für ihren Wahlkampf rund 32 Millionen Euro ausgaben, erhielten die 14 Parteien mit Anrecht auf Wahlkampfkostenerstattung circa 100 Millionen Euro staatliche Subventionen. ${ }^{27}$ Über den ganzen Zeitraum der Direktwahlen betrachtet lässt sich anhand der Ausgaben der SPD sogar ein Rückgang des Europawahlkampfbudgets ausmachen. Investierten die Sozialdemokraten 1979 noch circa 39 Millionen DM, waren es 1984 nur noch rund 27 Millionen DM beziehungsweise 1989 circa 32 Millionen DM. Im Jahr 1999 sanken die Europawahlinvestitionen sogar auf circa 20 Millionen DM. ${ }^{28}$

Mobilisierung im Wahlkampf: Aufgrund der abnehmenden Wahlbeteiligung bei Europawahlen erhielt die Mobilisierung der eigenen Stammwählerschaft für die Parteien zunehmend Priorität, während das Ansinnen, die wahlberechtigte Bevölkerung zu europolitisieren - beziehungsweise Einfluss auf europabezogene Einstellungen zu nehmen -, in den Hintergrund trat. Die Wahlkampfveranstaltungen, die im Rahmen von Europakampagnen initiiert wurden, sollten durch eine bunte und mediengerechte Aufmachung Aufmerksamkeit für die anstehenden Wahlen im Sinne einer Mobilisierungsstrategie wecken. Besonders deutlich trat die Kompensation fehlender europapolitischer Inhalte durch aufwändige Wahlkampfveranstaltungen im Rahmen der ersten und zweiten Direktwahl 1979 und 1984 zutage. ${ }^{29}$ Die Parteien griffen, wie in einem launischen Kommentar formuliert wurde, ,, weniger zu den Römischen Verträgen, als zu dem Erfolgsrezept der römischen Kaiser Brot und Spiele“. ${ }^{30}$ So ,tingelte ' beispielsweise die sozialdemokratische Spitzenkandidatin Katharina Focke in der heißen Wahlkampfphase 1984 mit einem Zirkus mit internationalem Spitzenprogramm durch die bundesdeutschen Städte. Ziel der Kampagne „Katharinas Zirkus. Eine Vorstellung von Europa" war es, die Bürger unterschwellig und unaufdringlich für Europa zu mobilisieren. ${ }^{31}$ Dieser Trend schwächte sich in den folgenden Jahren zwar ab, dennoch verzichtete

26 SPD, CDU, CSU, Bündnis 90/Die Grünen, FDP, PDS.

27 Vgl. Jens Tenscher: Mit halber Kraft voraus! Parteikampagnen im Europawahlkampf, in: Jens Tenscher (Hrsg.): Wahl-Kampf um Europa. Analysen aus Anlass der Wahlen zum Europäischen Parlament 2004, Wiesbaden 2005, S. 30-55, hier S. 39, insbesondere Tabelle 1, die unter anderem Bezug nimmt auf Daten von Jacques Gerstlé/Holli A. Semetko/Klaus Schoenbach/Marina Villa: The Faltering Europeanization of National Campaigns, in: Pascal Perrineau/Gérard Grunberg/Colette Ysmal (Hrsg.): Europe at the Polls: The European Elections of 1999, New York 2002, S. 59-77.

28 Siehe hier die entsprechenden Artikel aus der Westdeutschen Allgemeinen Zeitung, 06.06.1984, 20.06.1984, 01.06.1999.

29 Siehe zum Beispiel für 1979: Hans-Joachim Noack: Mobilisierungsversuche mit Freibier und Leberkäs. EuropaWahlkampf zwischen flau und lau: Mit großen Verschiebungen wird nicht gerechnet, abgedruckt in: Institut für Europäische Politik (Hrsg.): Die erste Direktwahl des Europäischen Parlaments. Motive, Wahlkampf, Resultate und Perspektiven, zusammengestellt und eingeleitet von Emanuel Richter, Bonn 1981, Dokument 48, S. 140-141.

30 Vgl. den Artikel „Europa-Wahlkampf zu Lande, zu Wasser und in der Luft“, in: Das Parlament 22/1984, S. 16.

31 Auch die anderen Parteien setzten 1984 auf symbolträchtige Kampagnen. Unter dem Motto „Wir brechen Bahn für Europa" reiste die FDP-Parteiprominenz in einem Sonderzug quer durch die Bundesrepublik, um in einzelnen Städten - in Begleitung von Jazzbands, Folkloregruppen etc. - Wahlwerbung zu betreiben. Die Christdemokraten gingen mit drei Schiffen auf,Europatörn“ und machten in über 150 Städten entlang des Rheins, des Mains und der Mosel Halt, um die „Überwindung von Entfernungen und Grenzen“ zu symbolisieren. Siehe hierzu auch Juliet Lodge: The 1984 direct elections to the European Parliament: a profile of the political forces, in: Journal of European Integration 1/1984, S. 33-57, hier S. 38. 
kaum eine Europawahlkampagne auf künstlerische Unterhaltungselemente wie beispielsweise Theater, Kabarett, Dichterlesungen und Musikshows. ${ }^{32}$

(Spitzen-)Kandidaten der Parteien: Die ersten Europawahlen 1979 stellten hinsichtlich der Kandidatenauswahl der Parteien eine Ausnahme dar. Gemäß der Gleichung „Prominenz = Zugkraft“ ${ }^{\text {33 }}$ zierten 1979 auffällig viele (ältere) Spitzenpolitiker und Verbandsfunktionäre die vordersten Plätze der Wahllisten - darunter für die SPD der ehemalige Bundeskanzler Willy Brandt (Listenplatz 1), der Vorsitzende des Deutschen Gewerkschaftsbundes (DGB) Heinz Oskar Vetter (Listenplatz 2), der ehemalige Ministerpräsident des Landes NordrheinWestfalen Heinz Kühn (Listenplatz 5) sowie der damalige Vorsitzende der deutschen Industriegewerkschaft Metall Eugen Loderer (Listenplatz 6). ${ }^{34}$ Der viel beschworene ,Opa ', der nach Europa entsendet wurde, fand vor dem Hintergrund dieser Rekrutierungsstrategie Eingang in zahlreiche Zeitungskommentare. Bereits für die zweite Europawahl 1984 ist aber eine deutliche Abweichung in der Kandidatenrekrutierung und Kampagnenführung der Parteien zu konstatieren: Der Bekanntheitsgrad der Spitzenkandidaten ließ merklich nach. Die Tendenz, auf die Nominierung prominenter Politiker als Kandidaten für das Europäische Parlament zu verzichten, setzte sich 1989 fort und folgte einem länderübergreifenden Trend. Die Aufstellung des damaligen belgischen Außenministers Leo Tindemans, des ehemaligen französischen Staatspräsidenten Valéry Giscard d'Estaing oder des früheren Ministerpräsidenten Laurent Fabius stellten hier Ausnahmen dar. Die Mehrzahl der Kandidaten rekrutierte sich fortan aus einem Reservoir jüngerer, weit weniger prominenter Politiker. ${ }^{35}$

Der Umstand, dass die deutschen Kandidaten für das Europäische Parlament - mit Ausnahme der ersten Direktwahl 1979 - weniger bekannt waren als die Parteieliten und nationalen Spitzenpolitiker wirkte sich auch medial aus. Selbst in der Europawahlberichterstattung waren die Kandidaten bei der Europawahl in geringerem Maße präsent als die Spitzenrepräsentanten der nationalen Parteien. Somit kann nur ein geringer Grad an medialer Personalisierung für die Europawahlen nachgewiesen werden. Eine Trendwende zeichnet sich hier erst bei der Wahl 2004 ab, bei der erstmals über die Europawahlkandidaten häufiger berichtet wurde, als über die nationalen Spitzenpolitiker. ${ }^{36}$

32 Siehe zur, Verbindung von Politik und Kunst“ auch die Beschreibungen der Wahlkampfveranstaltungen 1989 bei Stefan Reiser: Parteienkampagne und Medienberichterstattung im Europa-Wahlkampf 1989. Eine Untersuchung zu Dependenz und Autonomieverlust im Verhältnis von Massenmedien und Politik, Konstanz 1994, S. 113-114.

33 Vgl. den Bericht zum Wahlkampf in der Bundesrepublik von Helen Jannsen: Die heiße Phase steht noch bevor, abgedruckt in: Institut für Europäische Politik (Hrsg.): Die erste Direktwahl des Europäischen Parlaments. Motive, Wahlkampf, Resultate und Perspektiven, zusammengestellt und eingeleitet von Emanuel Richter, Bonn 1981, Dokument 47, S. 139-140.

34 Auch die CDU rüstete sich mit bekannten - aber im Vergleich zur SPD weniger prominenten - Spitzenkandidaten für die ersten europäischen Direktwahlen.

35 Vgl. auch Rudolf Hrbek: Das Europäische Parlament nach der Direktwahl 1989 - Reduzierte Handlungsfähigkeit durch größere Vielfalt?, in: integration 3/1989, S. 107-118 und Rudolf Hrbek/Carl-Christoph Schweitzer: Die deutschen Europa-Parlamentarier. Ergebnisse einer Befragung der deutschen Mitglieder des Europäischen Parlaments, in: Aus Politik und Zeitgeschichte B 3/1989, S. 3-18.

36 Vgl. Anne Wiorkowski/Christina Holtz-Bacha: Und es lohnt sich doch. Personalisierungsstrategien im Europawahlkampf, in: Christina Holtz-Bacha (Hrsg.): Europawahl 2004. Die Massenmedien im Europawahlkampf, Wiesbaden 2005, S. 174-196; Jürgen Wilke/Carsten Reinemann: Zwischen Defiziten und Fortschritten. Die Berichterstattung deutscher Tageszeitungen zu den Europawahlen 1979-2004, in: Jens Tenscher (Hrsg.): Wahl-Kampf um Europa. Analysen aus Anlass der Wahlen zum Europäischen Parlament 2004, Wiesbaden 2005, S. 157-176. Mit Blick auf den grundsätzlich geringen Personalisierungsgrad der Spitzenkandidaten sind jedoch auch Ausnahmen zu nennen. So kreiste der Europawahlkampf der rechtsextremen Republikaner 1989 fast ausschließlich um die Person ihres Bundesvorsitzenden Franz Schönhuber. Bei den Europawahlen 2004 avancierte hingegen die Spitzenkandidatin der FDP, Silvana Koch-Mehrin, zum Aushängeschild ihrer Partei. 
Medienberichterstattung: Als Hauptquelle für politische Informationen dienen für zahlreiche Bürger die Massenmedien. Europawahlen haben zwar, wie Frank Brettschneider und Markus Rettich dokumentieren, durchaus eine mediale „Katalysatorenfunktion“, diese zielt aber eher auf eine generelle Sensibilisierung für das Thema Europa als auf eine konkrete, wahlspezifische Berichterstattung. ${ }^{37}$ Der ersten Direktwahl 1979 kommt auch hier eine Sonderrolle zu. Das Medienecho auf den Europawahlkampf war - verglichen mit den nachfolgenden Wahlen zum Europäischen Parlament - beträchtlich. Für die Medien waren die ersten Europawahlen sowie die entsprechenden Kampagnen politische Ereignisse mit hohem Neuigkeits- und Nachrichtenwert, auch Dank der prominenten Kandidaten.

Für die beiden nachfolgenden Direktwahlen 1984 und 1989 ist hingegen schon in rein quantitativer Hinsicht ein deutlicher Rückgang in der TV-Berichterstattung zu verzeichnen. So ist beispielsweise die Anzahl der Beiträge zur Europawahl in den öffentlich-rechtlichen Sendern ARD und ZDF im Jahrzehnt zwischen 1979 und 1989 signifikant zurückgegangen. Dass sich der Umfang der Printmedienberichterstattung zu den Europawahlkämpfen - verglichen mit der Berichterstattung zu den Bundestagswahlkämpfen - seit 1979 auf einem vergleichsweise niedrigen Niveau stabilisiert hat, zeigen die umfassenden Untersuchungen von Jürgen Wilke und Carsten Reinemann. ${ }^{38}$ Demgegenüber macht Jeannette Döhner eine deutliche Steigerung der Berichterstattung im unmittelbaren Vorfeld der Wahlen 1994 und 1999 aus, betont aber, dass die Berichterstattung fast durchgängig mit ,einer negativen Wertung und Darstellung des Europäischen Parlaments unterlegt" wurde. ${ }^{39}$ Auffällig ist zudem, dass die Wahlkämpfe in der Medienberichterstattung bisher kaum transnationale Züge aufwiesen. Die meisten Medien haben die Europawahlen bisher lediglich aus nationaler Perspektive betrachtet, Berichte über die Wahlkämpfe in anderen EU-Mitgliedstaaten stellten kaum mehr als Randnotizen dar. Lediglich in der Qualitätspresse (Frankfurter Allgemeine Zeitung, Frankfurter Rundschau, Süddeutsche Zeitung, Die Welt) stieg die Anzahl von Beiträgen, die sich aus einer europäisch-vergleichenden Perspektive auf den Europawahlkampf bezogen, von 15 Prozent (1979) auf 24 Prozent (2004) leicht an. ${ }^{40}$

\section{Das Wahlsystem: sukzessive Harmonisierung}

Bereits im EGKS-Vertrag war die Möglichkeit einer Direktwahl der Abgeordneten (Art. 21 Abs. 3) vorgesehen, die in den Römischen Verträgen im Sinne des Auftrags zu einer Wahl mit einheitlichem Wahlverfahren präzisiert wurde (Art. 138 Abs. 3). ${ }^{41}$ Dieser konstitutionellen Vorgabe, die von den Abgeordneten des seinerzeit noch als Versammlung firmierenden Europäischen Parlaments wiederholt genutzt wurde, um Vorschläge zur Einführung von Direktwahlen einzubringen, ${ }^{42}$ stand jedoch der Ministerrat entgegen, der aufgrund der

37 Vgl. Frank Brettschneider/Markus Rettich: Europa - (k)ein Thema für die Medien, in: Jens Tenscher (Hrsg.): Wahl-Kampf um Europa. Analysen aus Anlass der Wahlen zum Europäischen Parlament 2004, Wiesbaden 2005, S. 136-156.

38 Vgl. Jürgen Wilke/Carsten Reinemann: Auch in der Presse immer eine Nebenwahl? Die Berichterstattung über die Europawahlen 1979-2004 und die Bundestagswahlen 1980-2002 im Vergleich, in: Christina Holtz-Bacha (Hrsg.): Europawahl 2004. Die Massenmedien im Europawahlkampf, Wiesbaden 2005, S. 153-173.

39 Jeannette Döhner: Die Europawahlen 1994 und 1999 in Deutschland. Eine Studie über die meinungsbildende Kraft der Printmedien und deren Einfluss auf das Wählerverhalten, Dissertation Universität Giessen 2005, S. 273.

40 Vgl. Wilke/Reinemann: Auch in der Presse immer eine Nebenwahl?, 2005, S. 166.

41 Der EGKS-Vertrag wurde mit Blick auf die Wahlen dem Wortlaut des EWG-Vertrages angepasst.

42 Bereits im Mai 1960 legte die Versammlung dem Ministerrat mit dem sogenannten Dehousse-Bericht einen ersten Entwurf vor, der - mit gewissen Übergangsfristen für einen Teil der Abgeordneten - Direktwahlen vorsah. Seit 1963 unterbreiteten auch einzelne nationale Parlamente Entwürfe zur nationalen Direktwahl der Abgeordneten, doch auch diese Bemühungen blieben erfolglos. Vgl. Grabitz/Läufer: Das Europäische Parlament, 1980, S. 204-208. Angesichts der Zurückhaltung des Rats hatte das Parlament 1969 und erneut 1972 sogar mit einer Untätigkeitsklage vor dem Gerichtshof gedroht. 
reservierten Haltung vor allem Frankreichs alle diesbezüglichen Anstrengungen der Parlamentarier ins Leere laufen ließ. Der Durchbruch erfolgte erst nach der französischen Präsidentschaftswahl 1974, als Valéry Giscard d'Estaing im Zuge der Institutionalisierung des Europäischen Rats den französischen Widerstand gegen Direktwahlen endgültig aufgab. Nachdem man sich aber auf die Einführung von Direktwahlen verständigt hatte, war der Vorrat an Gemeinsamkeiten aufgebraucht, so dass die Vereinbarung eines einheitlichen Wahlverfahrens, wie es in den Römischen Verträgen vorgesehen war, einer einvernehmlichen Lösung harrte. ${ }^{43}$ Der Berichterstatter des Europäischen Parlaments Schelto Patijn wollte die Konflikte umgehen, indem er für die erste Direktwahl zwar eine Harmonisierung des Wahltermins vorschlug, zugleich jedoch die Idee einbrachte, jedem Land zu erlauben, sein eigenes Wahlverfahren anzuwenden. Dieser Vorschlag ebnete letztlich den Weg zum Kompromiss. Der „Akt zur Einführung allgemeiner unmittelbarer Wahlen der Abgeordneten der Versammlung" sah vor, dass sich das Wahlverfahren in jedem Mitgliedstaat nach den innerstaatlichen Vorschriften bestimmt; ${ }^{44}$ im Gegensatz zum Entwurf des Parlaments wurde der Wahltermin aber auf einen viertägigen Zeitraum gestreckt. ${ }^{45}$

Die Wahlsysteme bei der ersten Europawahl orientierten sich dementsprechend an den Wahlen zu den nationalen Vertretungskörperschaften, lediglich Frankreich änderte den Grundtyp des Wahlsystems und wandte statt der absoluten Mehrheitswahl das Verhältniswahlsystem an. ${ }^{46}$ Die weiteren Details des Wahlsystems und -rechts unterschieden sich mit Blick auf Sperrklauseln, die Verfahren zur Zuteilung der Sitze und die Listenformen ebenfalls beträchtlich. ${ }^{47}$ Nach der ersten Direktwahl unterbreitete das Europäische Parlament erneut Vorschläge zur Vereinheitlichung des Wahlsystems. ${ }^{48}$ Der Berichterstatter, der französische EVP-Abgeordnete Jean Seitlinger, orientierte sich grob am Wahlsystem der Bundesrepublik und sah eine Verhältniswahl in Mehrpersonenwahlkreisen mit drei bis 15 Mandaten vor. Vor allem aufgrund des britischen Widerstands scheiterte der Entwurf jedoch ebenso wie ein 1985 vom CSU-Abgeordneten Reinhold Bocklet vorgestellter Entwurf, der ob seiner defensiven Natur bereits im Europäischen Parlament keine Mehrheit erhielt. Auf den 1993 eingereichten Vorschlag des Berichterstatters Karel De Gucht von den flämischen Liberalen reagierte der Rat ebenfalls nicht, obwohl hier als Zugeständnis an das britische Mehrheitswahlrecht die Möglichkeit vorgesehen war, in einzelnen Mitgliedstaaten zwei Drittel der Abgeordneten in Einmannwahlkreisen zu wählen.

Im Juli 1998 nahm das Europäische Parlament einen Vorschlag seines Berichterstatters, des griechischen EVP-Abgeordneten Georgios Anastassopoulos, an, der eine Verhältnis-

43 Vgl. Christoph Sasse et al. (Hrsg.): The European Parliament: Towards a uniform procedure for direct elections, Florenz 1981; Michael Steed: Twelve into one: The effect of using diverse procedures for the first European parliamentary elections, in: Roland Bieber/Albert Bleckmann/Francesco Capotorti u.a. (Hrsg.): Das Europa der zweiten Generation. Gedächtnisschrift für Christoph Sasse, Band 1, Baden-Baden 1981, S. 287310.

44 Akt zur Einführung allgemeiner unmittelbarer Wahlen der Abgeordneten der Versammlung, in: Amtsblatt der EG, Nr. L 278 vom 8. Oktober 1976, S. 5-11.

45 Die zögerlichen Vorbereitungsarbeiten Großbritanniens waren im Wesentlichen der Grund, dass die erste Direktwahl nicht wie vorgesehen 1978, sondern erst 1979 stattfand.

46 Vgl. als Überblick Eckhard Jesse: Wahlsysteme und Wahlrecht, in: Oscar W. Gabriel/Sabine Kropp (Hrsg.): Die EU-Staaten im Vergleich. Strukturen, Prozesse, Politikinhalte, Wiesbaden 2008, S. 299-322.

47 Während Belgien, Italien und Irland Wahlkreise entsprechend der Zahl der ihnen zustehenden Sitze einrichteten, Frankreich das ganze Land als Wahlkreis festlegte, erfolgten die Wahlen in der Bundesrepublik nach den Grundsätzen der Verhältniswahl auf Grundlage verbindbarer Landeslisten beziehungsweise einer Bundesliste.

48 Vgl. Francisco Aldecoa Luzarraga/Antonio Munoz Alvarez: Hacia una ley electoral uniforme para las elecciónes al Parlamento europeo: problemas que suscita, in: Revista de Instituciones Europeas 3/1986, S. 631-653; Christofer Lenz: Ein einheitliches Verfahren für die Wahl des Europäischen Parlaments. Unverwirklichte Vorgabe der Gemeinschaftsverträge, Baden-Baden 1995. 
wahl auf Grundlage von Listen vorsah. ${ }^{49}$ Dass bei der Europawahl 1999 - 20 Jahre nach der ersten Direktwahl - erstmals von einer gewissen Harmonisierung des Wahlsystems gesprochen werden konnte, war indes weniger auf das beharrliche Drängen des Europäischen Parlaments als vielmehr auf die veränderte Haltung der neuen britischen Regierung unter Tony Blair zurückzuführen. Diese verzichtete aufgrund allgemeiner Überlegungen zur Wahlreform in Großbritannien nach erbitterten Auseinandersetzungen mit dem Oberhaus darauf, das Mehrheitswahlrecht weiterhin zur Conditio sine qua non zu erheben und ermöglichte so, dass in allen Staaten nach dem Verhältniswahlsystem abgestimmt wurde, allerdings weiterhin mit erheblichen nationalen Unterschieden. ${ }^{50}$

Nachdem die Anpassung 1999 erstmals praktiziert worden war, wurde sie im Jahr 2002 auch rechtlich fixiert. In Abänderung des Akts von 1976 wurde einstimmig vereinbart, dass künftig die „Mitglieder des Europäischen Parlaments nach dem Verhältniswahlsystem auf der Grundlage von Listen oder von übertragbaren Einzelstimmen gewählt" werden. ${ }^{51}$ Die Frage der Wahlkreiseinteilung wurde in diesem Zusammenhang indes offen gelassen und auch der Wahltermin weiterhin flexibel gehandhabt, während hinsichtlich der Sperrklausel ein Quorum von maximal fünf Prozent fixiert wurde.

Mit Blick auf diese Parameter, die auch 2009 weiterhin Gültigkeit haben werden, kann die Auseinandersetzung um die Vereinheitlichung des Wahlverfahrens nicht als abgeschlossen betrachtet werden.52 Obgleich mit der Festlegung des Verhältniswahlrechts ein deutlicher Schritt in Richtung Harmonisierung vorgenommen wurde, bestehen unverändert erhebliche nationale Unterschiede. Diese werden sowohl von Seiten der Politik als auch von Vertretern der Wissenschaft als zentrale Hindernisse zur Demokratisierung der Europäischen Union betrachtet, weswegen die Reformdebatte weiter anhält. In jüngster Zeit war es der liberale britische Europaabgeordnete Andrew Duff, der mit dem Vorschlag, einen Teil der Abgeordneten über „halboffene länderübergreifende“ Listen zu wählen, die sich aus mindestens einem Viertel der Staaten zusammensetzen, Aufmerksamkeit erzielte. Würde dieser Vorschlag Realität, könnten die Wähler Kandidaten aus jedem Land wählen. Die Reaktionen in Parlament und Rat auf diesen Vorstoß haben jedoch deutlich gezeigt, dass für derart weitreichende Pläne gegenwärtig keine Mehrheit zu erzielen ist. ${ }^{53}$

49 Umstrittene Bereiche wie Sperrklauseln wurden hier als optionale Varianten behandelt. Regionale Wahlkreise sollten in den fünf größten Mitgliedstaaten errichtet werden. Dagegen sollten Mitgliedstaaten mit weniger als 20 Millionen Einwohnern nicht zur Errichtung regionaler Wahlkreise verpflichtet werden. Der Bericht schlug zudem vor, zu prüfen, ab 2009 zehn Prozent der Parlamentssitze im Rahmen eines europaweiten Wahlkreises zu vergeben.

50 In neun Staaten wurden bei den Wahlen 1999 die Kandidaten von einer nationalen Liste gewählt. Die anderen sechs Staaten teilten ihr Gebiet in vier bis elf Wahlkreise auf. In einigen Ländern gab es dabei starre Listen, deren Reihenfolge vom Wähler nicht beeinflusst werden konnte. Andere hatten lose gebundene Listen, bei denen die Reihenfolge vom Wähler abänderbar war. In Irland und Luxemburg konnten die Wähler sogar für Kandidaten unterschiedlicher Listen stimmen. Auch die Besetzung von frei gewordenen Mandaten unterschied sich: Während in zwölf Staaten der nächste Kandidat auf der Liste nachrückte, kamen in den anderen Ländern designierte Ersatzdelegierte zum Zuge.

51 Beschluss des Rates vom 25. Juni 2002 und 23. September 2002 zur Änderung des Akts zur Einführung allgemeiner unmittelbarer Wahlen der Abgeordneten des Europäischen Parlaments im Anhang zum Beschluss 76/ 787/EGKS, EWG, Euratom, in: Amtsblatt der EG, Nr. L 283 vom 21. Oktober 2002, S. 1-4.

52 David M. Farrell/Roger Scully: Electing the European Parliament: How Uniform are 'Uniform' Electoral Systems?, in: Journal of Common Market Studies 5/2005, S. 969-984.

53 Vgl. Europäisches Parlament: Entwurf eines Berichts über einen Vorschlag zur Änderung des Akts zur Einführung allgemeiner unmittelbarer Wahlen der Abgeordneten des Europäischen Parlaments vom 20. September 1976 (2007/2207(INI)), PE412.180v01-00 vom 17.09.2008, Berichterstatter Andrew Duff. 


\section{Die Wahlbeteiligung: anhaltender Rückgang}

Im Vorfeld des ersten europäischen Urnengangs 1979 hatte man aus einem ganzen Bündel von Erwartungen stets die Zielsetzung besonders hervorgehoben, mit dem Instrumentarium der Direktwahl „die subjektive Betroffenheit des Bürgers erhöhen zu können“ und somit auch „das Interesse und Engagement für die Gemeinschaftsentscheidungen und -prozesse zu verstärken“. ${ }^{54}$ Als Gradmesser für den Erfolg beziehungsweise die Grenze dieser Strategie wurde - gewissermaßen im Sinne eines Referendums über die grundsätzliche Zustimmung zum Integrationsprozess - die Wahlbeteiligung betrachtet. Bereits bei der ersten Direktwahl zeigte sich jedoch, wie fragil derartige Hoffnungen waren. Der allgemeine Mobilisierungsgrad blieb im Vergleich zu nationalen Parlamentswahlen - vielfach auch im Vergleich zu Regional- oder Kommunalwahlen - weit unter dem Durchschnitt. ${ }^{55}$ Bei den nächsten Wahlen verstärkte sich dieser Trend noch. Legt man die Gesamtwahlbeteiligung zugrunde, sank diese fünf Mal in Folge von einem Ausgangswert von 63 Prozent auf ein durchschnittliches Niveau von zuletzt 45,6 Prozent. ${ }^{56}$ Wie im Kontext der Wahlkampagnen und der Medienberichterstattung gezeigt wurde, kommt Wahlen zum Europäischen Parlament nach landläufigen Vorstellungen bei Parteien, Medien und in der Bevölkerung eine geringere Bedeutung zu als zu nationalen Parlamenten. Diese , less-at-stake‘ Perzeption hatte zur Konsequenz, dass die Bürger immer häufiger den Urnen fernblieben. Politiker wie Wissenschaftler stehen damit vor dem Paradoxon, erklären zu müssen, dass die Wahlbeteiligung von Wahl zu Wahl sank - obwohl die Kompetenzen des Europäischen Parlaments kontinuierlich ausgebaut wurden. ${ }^{57}$

Zwischen den einzelnen Mitgliedstaaten ergeben sich jedoch hinsichtlich der Wahlbeteiligung derart beträchtliche Unterschiede, dass grundsätzliche Feststellungen nur mit Vorsicht zu treffen sind.$^{58}$ Von der Forschung wird vielfach zwischen einzelnen Staatengruppen differenziert, die sich vor allem durch den Zeitpunkt ihres Beitritts zur Gemeinschaft unterscheiden. Bereits bei der ersten Europawahl 1979 lag in den Gründerstaaten Belgien (90,8 Prozent), Luxemburg (90,0 Prozent) und Italien (73,1 Prozent) die Beteiligung deutlich über dem EG-Durchschnitt - und blieb es in der Folge auch bei allen weiteren Wahlen. Dabei gilt aber zu berücksichtigen, dass in diesen drei Staaten eine Wahlpflicht besteht. In zwei weiteren Gründerstaaten der Gemeinschaft, in Deutschland und Frankreich, oszillierte die Wahlbeteiligung bei allen bisherigen Europawahlen um den Durchschnitt. Die Niederlande hingegen stellten stets das Schlusslicht unter den Gründungsmitgliedern dar, da die Wahlbeteiligung hier mit Ausnahme von 2004 rund 15 Prozentpunkte hinter der Deutschlands und Frankreichs lag.

54 Institut für Europäische Politik: Die erste Direktwahl, 1981, S. 15. Vgl. aber auch Karl-Heinz Naßmacher: Demokratisierung der Europäischen Gemeinschaften, Bonn 1972, S. 35-37.

55 Vgl. grundlegend für Deutschland: Markus Steinbrecher/Sandra Huber/Hans Rattinger: Turnout in Germany. Citizen Participation in State, Federal, and European Election since 1979, Baden-Baden 2007.

56 Die hier zugrunde gelegten statistischen Angaben beruhen auf Daten des Generalsekretariats des Europäischen Parlaments. Siehe zur grundsätzlichen Problematik zuverlässiger Daten zu den Europawahlen Markus Tausendpfund/Daniela Braun: Die schwierige Suche nach Ergebnissen der Wahlen zum Europäischen Parlament: Ein neuer Datensatz für die Wahlen 1979 bis 2004, in: Zeitschrift für Parlamentsfragen 1/2008, S. 84-93.

57 Vgl. Mikko Mattila: Why bother? Determinants of turnout in the European elections, in: Electoral Studies 3/2003, S. 449-468.

58 Siehe Jay G. Blumler/Anthony D. Fox: The Involvement of Voters in the European Elections of 1979: Its Extent and Sources, in: European Journal of Political Research 4/1980, S. 359-385; Jay G. Blumler/Anthony D. Fox: The European Voter: Popular responses to the first Community elections, London 1982; Hermann Schmitt/Renato Mannheimer: About voting and non-voting in the European elections of June 1989, in: European Journal of Political Research 1/1991, S. 31-54. 
In den Staaten der ersten Erweiterungsrunde 1973 pendelte die Wahlbeteiligung zumeist deutlich unter dem Durchschnitt; allein Irland wies hier mit durchschnittlich 55,5 Prozent höhere Werte auf. Großbritannien bildete bei vier von sechs Wahlen das nationale Schlusslicht bei der Wahlbeteiligung und lag mit seiner Partizipationsrate von durchschnittlich 33,3 Prozent deutlich unterhalb nationaler Kommunalwahlen. Die Staaten der Süderweiterung weisen hingegen höhere Werte als die Nordstaaten auf (Spanien durchschnittlich 58,3 Prozent und Portugal 47,5 Prozent), wobei im Falle Griechenlands (durchschnittlich 73,2 Prozent) die Wahlpflicht zu berücksichtigen ist. Die Staaten der dritten Erweiterungsrunde von 1995 kommen hingegen - grob betrachtet - auf ähnliche Werte wie die Staaten der Norderweiterungsrunde.

Während die 15 Mitgliedstaaten der, alten“ Europäischen Union bei den Wahlen 2004 noch eine durchschnittliche Beteiligungsquote von 46,8 Prozent erzielten, lag diese bei den zehn neuen EU-Mitgliedstaaten unter 30 Prozent, wobei auch hier der Mittelwert stark verzerrt. Die südeuropäischen Inselstaaten Zypern (71,2 Prozent) und Malta (82,4 Prozent) konnten eine Wahlbeteiligung vorweisen, die mit den Wahlpflichtstaaten der , alten ' Gemeinschaft konkurrierte. Am anderen Ende der Skala standen mittel- und osteuropäische Staaten wie Polen (20,87 Prozent) und Tschechien (28,3 Prozent). Das Schlusslicht bildete die Slowakei (16,9 Prozent), in der nicht einmal mehr jeder fünfte Stimmberechtigte sein Wahlrecht ausübte. ${ }^{59}$

\section{Die Wahlergebnisse: ,still second-order'?}

Die Abstrafung von nationalen Regierungsparteien gilt als wesentliches Kennzeichen von , second-order elections “ - mithin als Beleg für jenen Ansatz, dem bei allen bisherigen Europawahlen starke Erklärungskraft zugesprochen wurde. Es besteht eine hohe Wahrscheinlichkeit, so die vereinfachte Aussage des Modells von Hermann Schmitt und Karlheinz Reif, dass im Sinne einer taktischen Entscheidung des Wählers Regierungsparteien ebenso wie große Oppositionsparteien - abgestraft werden, während die eigene Stimme neuen kleinen Parteien oder radikaleren Protestparteien gegeben wird. Vor allem zur Mitte von nationalen Legislaturperioden wird dieser Sicht besondere Relevanz zugesprochen, womit die sinkende Popularität von Regierungsparteien zur zentralen Erklärungsvariablen von Europawahlergebnissen gemacht wird.

Die empirischen Trends der letzten 30 Jahre bestätigen das Modell in weiten Teilen: So deutete sich bereits bei der ersten Wahl an, dass die Bürger in der Europawahl eine willkommene Möglichkeit sahen, ihre Missbilligung gegenüber nationalen Regierungsparteien zum Ausdruck zu bringen. Mit Ausnahme der Niederlande - und somit in acht von neun Staaten der seinerzeitigen Europäischen Gemeinschaft - verloren die Regierungsparteien an Stimmen gegenüber den letzten nationalen Parlamentswahlen, zum Teil sogar deutlich, wie etwa die in Irland regierende Fianna Fáil, die eine schwere Niederlage erlitt. Zugleich zeichnete sich eine Stärkung kleinerer Parteien ab. Insgesamt entsandten 52 Parteien Abgeordnete ins erste Europäische Parlament, ${ }^{60}$ womit sich - entsprechend des Ansatzes der Sekundärwahlen - ein deutlicher Trend zur Differenzierung abzeichnete. ${ }^{61}$ In den ersten Wahlbetrachtun-

59 Zu den Europawahlen 2004 in den mittel- und osteuropäischen Mitgliedstaaten siehe Rudolf Hrbek (Hrsg.): European Parliament Elections 2004 in the Ten New EU Member States. Towards the Future European Party System, Baden-Baden 2005.

60 Nach dem Beitritt Griechenlands kamen fünf weitere Parteien hinzu.

61 Siehe Hrbek: Die EG nach den Direktwahlen, 1979; Herman/Hagger: The Legislation of Direct Elections, 1980; Juliet Lodge: The European Parliament after Direct Elections: Talking-Stop or Putative Legislature?, in: Journal of European Integration 3/1982, S. 259-284. 
gen wurde diesem Umstand jedoch nur begrenzte Aufmerksamkeit beigemessen. Von den zeitgenössischen Beobachtern wurde die erste Europawahl vor allem mit Blick auf die Frage analysiert, inwieweit sich eine Haltung für oder wider die europäische Integration im Wahlergebnis spiegelt. Eine deutliche Zustimmung zu europaskeptischen Positionen wurde dabei aber nur in Dänemark verzeichnet, wo die Volksbewegung gegen die Europäische Gemeinschaft 20,9 Prozent der Stimmen erzielte.

Bei den Wahlen 1984 war der Rekurs auf das ,second-order' Modell bereits weitgehend etabliert. So wurde wiederholt darauf verwiesen, dass in Deutschland die Grünen mit 8,4 Prozent der Stimmen ins Europäische Parlament einzogen, während die an der Bundesregierung beteiligten Liberalen den Einzug ins Parlament verfehlten. ${ }^{62}$ In einer Kurzanalyse der Wahl 1984 konstatierte Karlheinz Reif, dass es die nationalen Regierungsparteien sind, die bei den Europawahlen verloren haben - ,unabhängig von ihrer linken oder rechten, von ihrer eher pro-europäischen oder eher antieuropäischen Position“.63

Auch das Wahlergebnis 1989 wurde vor dem Hintergrund des ,typischen Nebenwahlcharakter[s] der Europawahl“ interpretiert. ${ }^{64}$ In Deutschland verloren die regierenden Unionsparteien an Stimmen, während die Republikaner mit 7,1 Prozent ins Europäische Parlament einzogen. Die SPD hatte als große Oppositionspartei von den Stimmverlusten der Regierungsparteien nicht profitieren können. In Frankreich erlitten die regierenden Sozialisten nach den Demonstrationen gegen die Schulgesetze der Regierung Mauroy deutliche Verluste, während die Grünen Stimmzuwächse verzeichneten und Front National sich konsolidierte. In Großbritannien gewann die oppositionelle Labour Party deutlich an Stimmen und auch in Portugal und Irland profitierte die Opposition. Es gab aber auch gegenläufige Beobachtungen: In Spanien behauptete sich die sozialistische Regierungspartei. ${ }^{65}$

Die Wahlen 1994 wurden als neuerliches Beispiel für die „Ersatzdramatik innenpolitischen Streits“ gewertet. ${ }^{66}$ Während in Deutschland die oppositionelle SPD im ,Superwahljahr 1994` es nicht vermochte, Erfolge zu erzielen, verloren die regierenden britischen Konservativen unter John Major deutlich. In Frankreich gewannen Kommunisten und Front National zusammen 18 von 87 französischen Mandaten, was als Plebiszit gegen die politische Klasse des Landes gewertet wurde. Ähnliches gilt für Belgien, wo die Rechtsradikalen deutlich zulegten, während die Regierungsparteien - Sozialisten und Christdemokraten erhebliche Einbußen zu verzeichnen hatten. Ähnliche Verluste beklagten auch Sozialdemokraten und Christdemokraten in den Niederlanden.

Bei der Europawahl 1999 bestätigte sich einmal mehr die Tendenz, Europawahlen als probates Mittel im Sinne eines, Denkzettels‘ zu instrumentalisieren. In Deutschland blieben

62 Rudolf Hrbek: Direktwahl 84: Nationale Testwahlen oder „,europäisches“ Referendum?, in: integration 3/1984, S. 158-166; Andrea Chiti-Batelli: Elezioni europee 1984 e riforma delle Comunità, Mailand 1984; Niels Sonntag/Kevin Featherstone: Looking towards the 1984 European Elections: Problems of Political Integration, in: Journal of Common Market Studies 3/1984, S. 269-282 sowie Lodge: The 1984 direct elections to the European Parliament, 1984

63 Karlheinz Reif: Nationale Regierungsparteien verlieren die Wahl zum Europäischen Parlament 1984, in: Zeitschrift für Parlamentsfragen 3/1984, S. 341-352.

64 Peter Gluchowski/Wolfgang Staudt/Ulrich von Wilamowitz-Moellendorff: Die dritten Direktwahlen zum Europäischen Parlament in der Bundesrepublik Deutschland, in: Aus Politik und Zeitgeschichte B 43/1989, S. 2551, hier S. 31. Siehe des Weiteren Juliet Lodge (Hrsg.): The 1989 Election of the European Parliament, Basingstoke 1990; Guillaume Devin: Les élections européennes de 1989: entre rétrospective et prospective, in: Pouvoirs 56/1991, S. 175-182; Institut für Europäische Politik (Hrsg.): Europawahl 1989 - Das Europäische Parlament im Spiegel der deutschen und internationalen Diskussion, eingeleitet und bearbeitet von Gieslint Grenz und Otto Schmuck, Bonn 1989.

65 Hrbek: Das Europäische Parlament nach der Direktwahl, 1989.

66 So Werner Weidenfeld: Die Bilanz der Europäischen Integration 1993/94, in: Werner Weidenfeld/Wolfgang Wessels (Hrsg.): Jahrbuch der Europäischen Integration 1993/94, Bonn 1994, S. 13-26, hier S. 13. 
die mittlerweile regierenden Sozialdemokraten überraschend deutlich hinter der Opposition zurück, allerdings gelang es der FDP als kleine Oppositionspartei abermals nicht, ins Europäische Parlament einzuziehen. In Großbritannien wurde die Europawahl zur ersten großen Niederlage der Labour-Regierung unter Tony Blair. Und auch in Griechenland verlor die sozialistische Regierungspartei. Dass dieser Trend allerdings erneut nicht für alle Staaten galt, zeigen andere Beispiele: In Frankreich behauptete sich die regierende Sozialistische Partei als stärkste Partei ${ }^{67}$ und in Spanien konnten die regierenden Konservativen ihren Wahlvorsprung zu den oppositionellen Sozialisten sogar deutlich ausbauen. Auch in Österreich siegte die regierende große Koalition, bestehend aus ÖVP und SPÖ, während die oppositionelle FPÖ unter Jörg Haider Stimmen abgeben musste.

Bei der Wahl 2004 hatten Regierungsparteien es erneut schwer, sich zu behaupten. Ungeachtet des parteipolitischen Lagers wurden in 19 von 25 Mitgliedstaaten die (großen) Regierungsparteien abgestraft, so etwa in Deutschland, England, Frankreich und Italien, aber auch in Polen, Tschechien, Ungarn oder in Lettland, Malta und Zypern. Allein den neu bestellten Regierungen der spanischen Sozialisten und der konservativen Nea Demokratia in Griechenland - sowie den Regierungsparteien in Luxemburg, Irland, Schweden und den Niederlanden - gelang es, sich zu behaupten oder an Stimmen noch zuzulegen. ${ }^{68}$

Bilanziert man die hier nur überblicksartig skizzierten Entwicklungen, so spricht einiges dafür, dem , second-order ${ }^{6}$ Modell eine unverändert hohe Erklärungskraft zuzusprechen. Zugleich deuten die letzten Europawahlen jedoch auch auf Differenzierungstrends hin. So hat die Anzahl der Staaten, in denen auch (große) Regierungsparteien als Sieger aus den Wahlen hervorgehen, zugenommen. Hieraus indes eine grundsätzliche Abkehr vom Modell der Sekundärwahl zu schließen, erschiene verfehlt. $\mathrm{Zu}$ verschieden sind die politischen, personellen und parteipolitischen Konstellationen und Rahmenbedingungen in den einzelnen Mitgliedstaaten, als dass sich aus den Differenzierungstrends ein eindeutiges neues Erklärungsmodell ableiten ließe.

\section{Fraktionsbildung: Stabilität statt Fragmentierung}

Als das direkt gewählte Europäische Parlament am 17. Juli 1979 zu seiner konstituierenden Sitzung zusammentrat, präsentierte es sich in weitgehend neuer Zusammensetzung. Lediglich 68 der 198 Abgeordneten des alten Parlaments waren in das neue, 410 Köpfe zählende Europäische Parlament zurückgekehrt. Trotz der im Vorfeld der Wahlen erheblich verstärkten transnationalen Parteiaktivitäten dauerte es aber einige Zeit, bis die Fraktionsbildung unter den direkt gewählten Vertretern von insgesamt 57 Parteien Kontur annahm. ${ }^{69}$ Ähnlich wie in den Folgejahren resultieren die Mehrheitsverhältnisse im Europäischen Parlament damit nicht allein aus den Wahlergebnissen, sondern zu einem guten Teil auch aus den Verhandlungen über die Zusammensetzung der Fraktionen. Hatten in den 1950er Jahren zunächst nur drei Fraktionen im Europäischen Parlament bestanden, ${ }^{70}$ vergrößerte sich diese Zahl im Vorfeld der Wahl 1979 auf sechs Fraktionen. ${ }^{71}$ Mit der Direktwahl erhöhte sich

67 Das rechte Lager in Frankreich war aufgrund von Unstimmigkeiten über die Europapolitik allerdings mit fünf unterschiedlichen Listen angetreten.

68 Vgl. hierzu auch Rudolf Hrbek: Europawahl 2004: neue Rahmenbedingungen - alte Probleme, in: integration 3/2004, S. 211-222.

69 Die drei Westberliner Abgeordneten waren nicht direkt, sondern durch das Abgeordnetenhaus gewählt worden.

70 Als Quorum für den Fraktionsstatus wurde zunächst eine Mindestzahl von neun Delegierten (= 12 Prozent) festgesetzt.

71 Die Gaullisten (1965), nordischen Konservativen (1973) und Kommunisten (1973) hatten ebenfalls Fraktionen gebildet. Erleichtert wurde die Fraktionsbildung durch Änderungen der Geschäftsordnung. Im Oktober 1973 war festgelegt worden, dass zur Bildung einer Fraktion lediglich 10 von 183 Abgeordneten (= 5 Prozent) notwendig waren. 
diese Zahl - trotz der , second-order ${ }^{6}$ Effekte - jedoch nicht substanziell auf insgesamt sieben Fraktionen.

Obwohl die Zahl der Parlamentarier von 410 im Jahr 1979 bis auf zeitweilig 785 Mitglieder (nach dem Beitritt Bulgariens und Rumäniens 2007) anstieg und mittlerweile Abgeordnete von 177 nationalen Parteien im Europäischen Parlament sitzen, ist es nicht zu der vielfach befürchteten Fragmentierung im Europäischen Parlament gekommen. Hatte sich anfänglich noch ein gewisser Trend zur Ausweitung der politischen Gruppierungen abgezeichnet - in der konstituierenden Sitzung des Europäischen Parlaments im Jahre 1984 waren es acht und nach den Wahlen 1989 sogar zehn Fraktionen ${ }^{72}$ - ist diese Schwelle seitdem nicht überschritten worden, sondern die Anzahl der Fraktionen sogar rückläufig. In der Wahlperiode 1994-1999 konstituierten sich neun, nach den Europawahlen 1999 acht Fraktionen. Dieser Konzentrationsprozess ist im Wesentlichen auf die ,Schirmpolitik ' der EVPED Fraktion zurückzuführen, die sowohl die zuvor eigenständige konservative Fraktion der „Europäischen Demokraten“ (unter anderen britische, spanische und dänische Konservative) als auch die „Sammlungsbewegung der Europäischen Demokraten“ (unter anderen französische Gaullisten) unter dem neuen gemeinsamen Dach vereinte. Aber auch die Novellierung der Geschäftsordnung des Europäischen Parlaments trug entscheidend zur Konzentration bei. Bereits 1999 wurde die Bildung einer Fraktion aus einem einzigen Mitgliedstaat abgeschafft. In $\S 29$ der ab Juli 2004 geltenden Geschäftsordnung wurde festgeschrieben, dass die Mitglieder einer Fraktion künftig in mindestens einem Fünftel der Mitgliedstaaten gewählt sein müssen.

In der Wahlperiode 2004 konstituierten sich dann sogar nur noch sieben vergleichsweise stabile Fraktionen. ${ }^{73}$ Auffällig ist dabei die Tendenz zu ,Fraktionsallianzen“. Bereits nach der Erweiterungsrunde 1995 hatte sich die „Konföderale Fraktion der Vereinigten Europäischen Linken/Nordische Grüne Linke“ (GUE/NGL) gebildet, die sowohl kommunistische Parteien aus Südeuropa als auch linke Parteien aus Skandinavien umschloss. Seit 1999 wird von den europäischen Grünen und den in der Europäischen Freien Allianz vereinten Regionalparteien das Experiment praktiziert, zwei unterschiedliche europäische Parteiorganisationen im Rahmen einer Fraktion des Europäischen Parlaments zusammenzuschließen. Nach den Wahlen 2004 griffen auch die Liberalen und Zentristen dieses Modell auf. Die auf europäischer Ebene als „Europäische Liberale Demokratische und Reformpartei“ firmierenden Liberalen kooperieren mit der im April 2004 gegründeten „Europäischen Demokratischen Partei“ der Zentristen gemeinsam in der ALDE-Fraktion. Es gehört mit zu den spannendsten Fragen, ob diese Tendenz zur Konzentration und Kooperation im Europäischen Parlament weiter anhält. Angesichts der zahlreichen Anreize, die mit dem Fraktionsstatus verbunden sind - so etwa die Beantragung von Debatten, die Verteilung von Redezeiten, die Vertretung in den Präsidien der parlamentarischen Ausschüsse und die Vergabe von Büros und Sekretariatskräften - spricht einiges dafür, zumal die Hürde zur Fraktionsbildung nochmals erhöht wurde. Nach der Europawahl 2009 soll ein Minimum von 25 Abgeordneten aus mindestens einem Viertel - statt einem Fünftel - der Mitgliedstaaten zur Bildung einer Fraktion erforderlich sein. ${ }^{74}$ Auf der anderen Seite sind bei Weitem aber nicht alle Fraktionszusammen-

72 Dies unter anderem weil sich die nicht-sozialdemokratische Linke auf zwei Fraktionen aufteilte.

73 Das Experiment der 2007 etablierten rechtsextremen Fraktion „Identität, Tradition, Souveränität“ (ITS) blieb aufgrund des Austritts der Abgeordneten der Großrumänien-Partei eine Episode.

74 Siehe die Geschäftsordnung des Europäischen Parlaments in der Fassung vom 9. Juli 2008. Vgl. Europäisches Parlament: Beschluss des Europäischen Parlaments vom 9. Juli 2008 zur Änderung von Artikel 29 der Geschäftsordnung des Europäischen Parlaments - Bildung der Fraktionen (2006/2201(REG)), P6_TA(2008)0351 vom 09.07.2008. 
schlüsse Wunschpartnerschaften, sondern vielmehr Zweckgemeinschaften. So tragen sich vor allem die britischen Konservativen seit Langem mit Plänen, (wieder) eine eigenständige konservative Fraktion im Europäischen Parlament zu etablieren. Entsprechende Überlegungen wurden im März 2009 als Wahlkampfargument publik gemacht, zumal auch die tschechische ODS mit dem EU-skeptischen Präsidenten Václav Klaus Mitte März 2009 bekundete, sich nicht mehr an der EVP-ED-Fraktion beteiligen $\mathrm{zu}$ wollen. Und auch die (europaskeptischen) Nordischen Grünen haben deutlich gemacht, dass eine Fortsetzung ihrer Fraktionsallianz mit der Europäischen Linken nicht zwingend ist.

\section{Die Europawahlen zwischen Kontinuität und Wandel: eine Standortbestimmung}

Bilanziert man abschließend die Beobachtungen in den hier näher beleuchteten Untersuchungsfeldern, so sind mit Blick auf die Europawahlen der letzten 30 Jahre sowohl Anzeichen für Kontinuität als auch für Wandel festzustellen. Die empirischen Befunde zeugen zwar von der dominierenden Persistenz des Sekundärwahlmodells, sie lassen zugleich aber auch Entwicklungslinien in Richtung Europäisierung erkennen: Obwohl die Medienpräsenz europäischer Themen allgemein zugenommen und auch das Europäische Parlament in der Wahrnehmung von Öffentlichkeit und Politik an Bedeutung gewonnen hat, wirkte sich dieser Trend bisher nicht in gleichem Maße auf die Direktwahlen aus. Sieht man von der ersten Wahl $1979 \mathrm{ab}$, der angesichts ihres Neuigkeitswertes eine Sonderrolle zukommt, blieb die Medienberichterstattung bei allen weiteren Europawahlen begrenzt; nennenswerte Veränderungen sind in der Zeitachse nicht auszumachen. Allein die wachsende Aufmerksamkeit, die den Spitzenkandidaten zugebilligt wird, und die behutsame Vereinheitlichung des Wahlauftritts in transnationaler Hinsicht deuten in jüngerer Zeit auf einen gewissen Wandel hin. Zugleich zeichnen sich hinsichtlich der Kampagnen aber weder bei den unvermindert national geprägten Wahlkampfthemen noch bei der schwach profilierten Wahlkampforganisation einschließlich knapper Kampagnenzeiträume, schmaler Budgets und eines geringen Grades an Externalisierung wahlkampfspezifischer Aufgaben - grundlegende Änderungen ab.

Demgegenüber weist die Entwicklung der Wahlsysteme eine Tendenz zur Anpassung auf. Die EU-weite Anwendung des Verhältniswahlrechts seit 1999 und dessen rechtliche Verankerung seit 2002 lassen einen grundsätzlichen Trend zur Harmonisierung erkennen. Es bleibt dem Standpunkt des Betrachters überlassen, ob diese Anpassung eher als Minimalkonsens oder als wichtige Etappe auf dem Weg zur Vereinheitlichung zu interpretieren ist. Alle darüber hinausgehenden Reformanstrengungen in Bezug auf Wahldatum, Listenform etc. scheinen gegenwärtig jedoch ebenso wenig konsensfähig zu sein wie die Wahl einer begrenzten Anzahl von Parlamentariern über europäische beziehungsweise europaweite Listen. Damit bleibt ein seit den 1950er Jahren eingehend diskutiertes Thema auf absehbare Zeit in der Schwebe, während andere, lange Zeit kontrovers erörterte Problemfelder - so etwa der rechtliche Status der Parteien und der Abgeordneten - in den letzten Jahren im Sinne einer Harmonisierung der rechtlichen Strukturen auf europäischer Ebene geklärt wurden. ${ }^{75}$

Der kontinuierlich zurückgehenden Wahlbeteiligung wird aus demokratietheoretischer Sicht beträchtliche Aufmerksamkeit beigemessen. Obgleich die Beteiligungsquote vor allem

75 Vgl. Thomas Jansen/Justus Schönlau: Der Parteienartikel als Impulsgeber? Die Entwicklung der Rechtsgrundlagen für die „Europäischen Politischen Parteien“, in: Jürgen Mittag (Hrsg.): Politische Parteien und europäische Integration. Entwicklung und Perspektiven transnationaler Parteienkooperation in Europa, Essen 2006, S. 515-534 und Jürgen Mittag: Wegmarke für die Parlamentarisierung der Europäischen Union: Die finanziellen Neuregelungen des europäischen Abgeordnetenstatuts, in: Zeitschrift für Parlamentsfragen 4/2006, S. 713728. 
entlang der Spannungslinie, alte versus jüngere Mitgliedstaaten“ erhebliche Differenzen aufweist, ist in fast allen Mitgliedstaaten ein Rückgang auszumachen. Damit steht einer grundsätzlichen diffusen Unterstützung für die Europäische Union, die auch von zahlreichen Eurobarometerumfragen dokumentiert wird, eine spezifische Verweigerung dieser Unterstützung bei Europawahlen gegenüber. Vor diesem Hintergrund stellt das wachsende Bemühen um eine Mobilisierung für Europawahlen ebenfalls einen wichtigen Trend der beiden letzten Dekaden dar.

Die Wahlergebnisse zeugen mit Blick auf das dominante politikwissenschaftliche Paradigma der , second-order elections ‘ ebenfalls eher von Kontinuität, denn von Wandel. Allgemeine Wahlbetrachtungen zeigen ebenso wie Detailstudien, dass Auswirkungen auf nationale Wahlen und die innerstaatliche Machtverteilung vielfach im Zentrum der Auseinandersetzungen von Europawahlen stehen. Die Entscheidung zum Gang an die europäischen Wahlurnen wird damit im Wesentlichen vom Vertrauen beziehungsweise Misstrauen gegenüber der nationalen Regierung geprägt und nicht von europäischen Themen. Mit den Erweiterungsrunden und einer immer größeren Zahl von Mitgliedstaaten deutet sich jedoch eine wachsende Pluralität und damit eine zunehmende Abweichung vom generellen Trend an, die nicht zuletzt auf die unterschiedlichen politischen Systeme der EU-Mitgliedstaaten, deren Traditionen und Parteiensysteme, aber auch auf die Interaktion im Mehrebenensystem zurückzuführen ist. Die wissenschaftliche Forschung hat hierauf bereits mit verschiedenen Weiterentwicklungen des , second-order ${ }^{6}$ Modells reagiert, ${ }^{76}$ in denen unter anderem davon ausgegangen wird, dass nicht mehr nur nationale Regierungsparteien abgestraft werden, sondern „Europas Wähler verstärkt dazu übergegangen sind, europapolitische, insbesondere europaskeptische Signale an die heimischen Parteien zu senden “. ${ }^{77}$

Ungeachtet einer Verdreifachung der Zahl nationaler Parteien im Europäischen Parlament zwischen 1979 und 2004 zeugen die aus den Europawahlen indirekt resultierenden Fraktionsbildungsprozesse von einer bemerkenswerten Stabilität. Nicht zuletzt aufgrund des Instrumentariums der Geschäftsordnung, mit dem die Anforderungen an die Bildung einer Fraktion im Europäischen Parlament verschärft wurden, ist die Anzahl der Fraktionen des Europäischen Parlaments seit der ersten Direktwahl weitgehend konstant geblieben. Inwieweit das ebenfalls an Bedeutung gewinnende - und bisher kaum näher untersuchte - Muster der Fraktionsallianzen hier als dauerhaftes Strukturmerkmal oder eher als temporäre Erscheinung betrachtet werden kann, ist gegenwärtig noch nicht abzusehen.

$\mathrm{Ob}$ - und in welchem Maße - die Reformdebatten der letzten Jahre Einfluss auf die Europawahlen haben werden, ist wohl erst bei einer späteren Europawahl abzusehen. Gegenwärtig wird vor allem den geplanten Reformen der institutionellen Rahmenbedingungen Bedeutung zugemessen, da die Vorgabe, die Ergebnisse der Europawahl bei der Nominierung des Kommissionspräsidenten zu berücksichtigen, auf eine Personalisierung beziehungsweise Politisierung des Wahlkampfs hinwirken kann. Relevant könnten aber auch die jüngsten Reformen bei den Organisationsstrukturen der Parteien auf europäischer Ebene werden, die diesen nicht nur größere finanzielle Spielräume, sondern auch erweiterte Aktionsmöglichkeiten im Wahlkampf eröffnen. Ob Reformen beziehungsweise eine weitere

76 Vgl. etwa Cliff Carrubba/Richard J. Timpone: Explaining Vote Switching Across First- and Second-Order Elections. Evidence from Europe, in: Comparative Political Studies 3/2005, S. 260-281 oder Philip Manow/ Holger Döring: Electoral and Mechanical Causes of Divided Government in the European Union, in: Comparative Political Studies 10/2008, S. 1349-1370.

77 Vgl. hierzu etwa Philip Manow: Elektorale Effekte negativer Integration? Die Europäische Gemeinschaft und die Europawahlen, 1979 bis 2004, in: Jens Beckert/Bernhard Ebbinghaus/Anke Hassel/Philip Manow (Hrsg.): Transformationen des Kapitalismus, Frankfurt am Main 2006, S. 337-357. 
Harmonisierung der Wahlsysteme zu einer stärkeren Europäisierung der Wahlen führen und so möglicherweise auch zur Stärkung des Europäischen Parlaments beitragen wird bleibt abzuwarten, ${ }^{78}$ da diesen Forderungen die Vorstellung eines pluralen Europas entgegensteht, das die Existenz unterschiedlicher, aus den jeweiligen Traditionen politischer Repräsentation gespeister Wahlsysteme anerkennt. ${ }^{79}$

Ein wichtiger Strang künftiger Europawahlforschung dürfte darin bestehen, die hier lediglich ausschnittartig zusammengefassten Forschungsergebnisse und grob skizzierten Entwicklungsstränge in detaillierteren Studien mit einem erweiterten Set von Kategorien und Variablen zu untersuchen. Eine stärker diachron orientierte Perspektive und der Rekurs auf eine Zusammenschau der Erträge der einzelnen integrationswissenschaftlichen Disziplinen vermag dabei sowohl allgemeine Trends als auch spezifische Sonderheiten deutlicher zu konturieren, als dies in den bisherigen Detailbetrachtungen der Fall war. Eine produktive Verknüpfung sozialwissenschaftlicher Ansätze mit den Fragestellungen, Methoden und Quellen von Juristen, Historikern und Medienwissenschaftlern scheint dabei unumgänglich, will man den Europawahlen als ein Schlüssel zur Analyse des Europäischen Parlaments im Besonderen und der europäischen Integration im Allgemeinen gerecht werden.

78 Vgl. in diesem Sinne Simon Hix: What's Wrong With the European Union \& How to Fix It, Cambridge 2008.

79 Siehe Dieter Nohlen: Wie wählt Europa? Das polymorphe Wahlsystem zum Europäischen Parlament, in: Aus Politik und Zeitgeschichte B 17/2004, S. 29-37. 